\title{
Latest Publications in Cytometry Part A You Should Not Miss
}

\author{
Selected by Attila Tárnok, Leipzig, Germany
}

\section{Measuring Bad Iron}

Proper iron supply is important for health; deficiency or excess are involved in diseases (e.g., iron-deficient anemia or ironoverload, respectively). Although most iron is firmly bound (e.g., in hemoglobin), some - the labile iron pool (LIP) - is more loosely bound to various ligands. The latter iron is involved in generation of oxidative radicals and their deleterious consequences. The level of LIP in cells is regulated to meet their requirements for iron but prevent excess. Prus and Fibach describe a multi-parameter flow cytometry procedure for measuring LIP in human blood cells. Since flow cytometry is a standard methodology in many clinical laboratories, the novel procedure has potential for evaluating LIP in various diseases and for determining the efficacy of iron-chelation therapy.

Prus E, Fibach E: Flow cytometry measurement of the labile iron pool in human hematopoietic cells. Cytometry A 2008;73A (1):22-27. DOI: 10.1002/cyto.a.20491

\section{Human Sperm DNA Fragmentation Results: Technique Dependent}

The TUNEL assay is a technique used to evaluate the DNA fragmentation in human sperm. The results of the technique can be evaluated using flow cytometry or optical microscopy but are usually reported using only one of the two methods. However, the results obtained using each of these two approaches are different. Thus, the comparison of the results described in the different articles has so far been difficult. Domínguez-Fandos and co-workers applied these two approaches in the same human sperm samples. Although they detected a clear positive correlation in the TUNEL results as measured by the two techniques, the percentage of TUNEL positive spermatozoa detected using flow cytometry was higher as compared to that detected in optical microscopy. This work should be valuable to compare the results described in independent TUNEL studies using either optical microscopy or flow cytometry.

Domínguez-Fandos D, et al: Human sperm DNA fragmentation: Correlation of TUNEL results as assessed by flow cytometry and optical microscopy. Cytometry A 2007;71A(12): 1011-1018. DOI: 10.1002/cyto.a.20484

\section{Interpreting the Sugar Code of Dying Cells}

Death is not the final destiny of cells in multicellular organisms: the corpses must be cleared by neighboring-cell or professional phagocytes. During this process moribund cells have the last chance to attract attention: they signal all-clear or danger, provoking anti-inflammation and tolerance or inflammation and immune response, respectively. These signals are 'interpreted' by various soluble adaptor proteins and surface receptors. However, the molecular nature of the 'translators' and the 'signals' is not yet fully identified. Based on the emerging concept of the sugar code, Beer and co-workers reasoned that cell surface glycans and endogenous tissue lectins are involved in this communication. Indeed, they revealed specific interaction processes by systematically testing this hypothesis using human lectins of the galectin family.

Beer A, et al: Human galectins as sensors for apoptosis/necrosis-associated surface changes of granulocytes and lymphocytes. Cytometry A 2008;73A(2):139-147.DOI: 10.1002/ cyto.a. 20510

\section{Statistical Analysis of Data on Reactive Oxygen Species (ROS)}

ROS are continuously produced during cellular metabolism in all aerobic organisms. Small amounts of ROS are indispensable in several biochemical processes, however high doses and/or inadequate removal of ROS result in oxidative stress, which can lead to cell death and tissue injury. Flow cytometry offers several advantages in measurement of ROS, mainly the 
ability to quantitatively examine a large number of individual cells in a very short time. Nevertheless, even if this technique is widely used, there is no agreement on the statistical analysis of cytometric data. In their current work, Brescia and Sarti investigate the critical, however in most cases neglected, question of analyzing flow cytometry ROS data. They introduce a new algorithm (a modification of the Lampariello procedure) and show its superiority over the available approaches.

Brescia F, Sarti M: Modification to the Lampariello approach to evaluate reactive oxygen species production by flow cytometry. Cytometry A 2008;73A(2):175-179. DOI: 10.1002/cyto.a. 20508

\section{Membrane Potential of Individual Mitochondria}

Mitochondrial ATP production by oxidative phosphorylation is crucial for cellular energy homeostasis and requires a sufficiently negative potential $(\Delta \psi)$ across the mitochondrial inner membrane. Alterations of this parameter are critically involved in human disease, including inborn errors of metabolism, agerelated neurological diseases, cardiovascular disease, cancer and diabetes. Distelmaier and co-workers present a novel life cell assay combining tetramethyl rhodamine methyl ester (TMRM) staining and videomicroscopy with automated image processing to assess $\Delta \psi$ in living human skin fibroblasts at the level of individual mitochondria. Moreover, combined fluorescence microscopy of TMRM and a mitochondria-targeted green fluorescence protein (mito-AcGFP1) allowed quantification of $\Delta \psi$ under conditions of severe mitochondrial depolarisation. This approach will be useful to determine alterations in $\Delta \psi$ in fibroblasts of patients with mitochondrial disease.

Distelmaier F, et al: Life cell quantification of mitochondrial membrane potential at the single organelle level. Cytometry A 2008;73A(2):129-138. DOI: 10.1002/cyto.a.20503

\section{Multiparameter Cell Cycle Analysis of Acetone Treated Cells}

The incompatibility of fixation/permeabilization methods optimal for both DNA content analysis and surface/internal immunofluorescences is a persistent problem in flow cytometry. Methods that result in stoichiometric DNA labeling (e.g., ethanol fixation) generally damage cell epitopes and/or fluorochrome conjugated antibodies, while crosslinking fixation (e.g., paraformaldehyde) that can preserve epitopes, fluorochromes and antibodies inhibits stoichiometric binding of DNA dyes. Carbonari and co-workers describe a novel method, termed FAST (Flow Acetone-Staining Technique), for multiparameter DNA flow cytometry which offers several advantages over previously described methods. These include simplicity, reproducibility and, because cell morphology is very well preserved, immunophenotypic characterization of cells can be done by multi-color fluorescence, thus DNA staining is of excellent quality.

Carbonari M, et al: Flow acetone-staining technique: A highly efficient procedure for the simultaneous analysis of
DNA content, cell morphology, and immunophenotype by flow cytometry. Cytometry A 2008;73A(2):168-174. DOI: 10.1002/cyto.a.20521

\section{Electronic Volume of Stem Cells}

Measurement of electronic cell volume by the Coulter Principle is more accurate than use of forward angle light scatter in a flow cytometer. In the present report, Sharma and co-workers have used a Beckman Coulter Quanta Flow analyzer to determine the mean electronic cell volume and diameter of normal human peripheral blood cells and hematopoietic progenitor cells obtained by apheresis (HPC-A) from patients with hematological malignancies. Samples after erythrocyte lysis or Ficoll Hypaque sedimentation were stained with CD45-FITC and PE-labeled CD34, CD90, CD117 or CD133 antibodies and analyzed for electronic volume and two color fluorescence. CD90 positive cells had the smallest mean electronic volume while cells with CD133, CD117, CD34 and CD45 were increasingly larger in volume. Correlation of cell volume with stem cell marker expression may allow for the identification of small stem cells, which may not express the conventional markers used for the identification of stem cells in HPC-A samples.

Sharma S, et al: Cellular volume and marker expression in human peripheral blood apheresis stem cells. Cytometry A 2008;73A(2):160-167. DOI: 10.1002/cyto.a.20524

\section{The Benefit of a Multiparametric Approach to Defining Translational Animal Models}

One of the more powerful aspects of polychromatic flow cytometry is the ability to simultaneously evaluate lineage and function in heterogenous cell populations on a cell by cell basis. In the current offering from Capocasale and co-workers, panels of well established lineage markers (Ter-119, Gr-1, CD11b, Sca-1 and IL-7R ) were combined with function phenotype markers for activation, apoptosis, and cell death (CD71, annexin-V and 7-AAD) and cell cycle markers (BrdU and 7-AAD) in a novel way. This was done to study the bone marrow of human TNF- $\alpha$ transgenic mice. When coupled with conventional hematology, histopathology and serum cytokine measurements, the polychromatic cytometric analysis allowed characterization of these mice as a clinically relevant model of myelodysplasia and anemia of chronic disease. Moreover, this work demonstrates how a multidisciplinary approach can extend the usefulness of established animal models of disease.

Capocasale RJ, et al: Myelodysplasia and anemia of chronic disease in human tumor necrosis factor- $\alpha$ transgenic mice. Cytometry A 2008;73A(2):148-159. DOI: 10.1002/cyto.a.20512

\section{Cytoplasmic Clotting Factor in Studying Leukemias}

Detection of phenotypic aberrancies are crucial in the followup of leukemic patients. Kiss and co-workers summarize the role of blood coagulation factor XIII subunit A (FXIII-A) in the coagulation cascade and the expression of the molecule in 
normal cells. In addition they point out the benefit of identifying FXIII-A not only in the malignancies of the mono- and megakaryocytic cell lineages (i.e. myeloblastic leukemia), but also in cases of lymphoblastic leukemia, rendering it a valuable diagnostic tool in the investigation of all acute leukemias. Kiss F, et al: A coagulation factor becomes useful in the study of acute leukemias: studies with blood coagulation factor XII. Cytometry A 2008;73A(3):194-201. DOI: 10.1002/ cyto.a. 20485

\section{FISHing with Cross-Hairs}

Amongst cell-based markers for the detection of bladder cancer, the UroVysion ${ }^{\mathrm{TM}}$ FISH (fluorescent in situ hybridization) test has proven to be a powerful tool for detecting tumor cells in voided urine. The test has a high specificity and a relatively high sensitivity however this diagnostic method carries uncertainties: The target cells are obviously exfoliated uroepithelial cells but urine does not only contain these cell types. Claiming atypia (the first step according to protocol), and hence urothelial origin based solely on DAPI morphology might be deceptive and misleading during evaluation. Non-uroepithelial cells with normal chromosomal garniture and those - due to false positives - that show aberrations could both distort results. Preselection of uroepithelial cells could circumvent these distortions and hasten evaluation at the same time. In the method presented by Pajor and co-workers, an automated microscope carries out both - identification of CK-7 positive cells and relocation during consecutive FISH - automatically. Their procedure combines chromogenic immunophenotyping and FISH, allowing the target-specific evaluation of Uro Vysion $^{\mathrm{TM}}$.

Pajor G, et al: Increased efficiency of detecting genetically aberrant cells by UroVysion test on voided urine specimens using automated immunophenotypical preselection of uroepithelial cells. Cytometry A 2008;73A(3):259-265. DOI: 10.1002/cyto.a. 20528

\section{D Mapping of Anisotropy}

A new tool has become available for mapping the anisotropy in microscopic samples. Probably all biological tissues or cells, due to their highly organized nature, contain anisotropic microscopic regions. They might exhibit linear or circular dichroisms and fluorescence emission anisotropies, which originate from ordered molecular arrays; they might also be birefringent and might contain local variations in the microviscosity and thus in P, the degree of polarization of fluorescence emission. All these quantities, which carry unique information on the molecular organization of the sample, can now be determined in a recently assembled DP-LSM that uses differential polarization attachments similar to those in dichrographs. Steinbach and co-workers show that confocal imaging of fluorescence detected linear dichroism of plant cell walls, stained with intercalating dyes, yield high precision data suitable for structural models, while Gombos and co-workers uses P-imag- ing to confirm the microdomain organization in the plasma membranes of live immune cells.

Steinbach $G$, et al: Imaging fluorescence detected linear dichroism of plant cell walls in laser scanning confocal microscope. Cytometry A 2008;73A(3):202-208. DOI: 10.1002/ cyto.a. 20517

\section{Showdown with Multi-Molecular Complexes by tsFRET}

Dysregulation of signal transduction processes starting from assemblies of cell surface molecules contributes to the pathogenesis of various cancers. Interactions between members of multi-molecular signaling complexes are therefore of particular interest. Fazekas and co-workers now implement a new, innovative experimental set-up termed two-sided FRET (tsFRET) for assessing molecular interactions of up to three distinct species. Correlating two pairwise interactions of three arbitrarily chosen molecules, they report anti-correlation between $\beta 1$-integrin - ErbB2 heteroassociation and ErbB2 homoassociation on trastuzumab sensitive cells, and observe markedly higher FRET efficiency values of $\beta 1$-integrin ErbB2 heteroassociation at the focal adhesion regions of attached cells. By means of tsFRET, they deduce that the homoassociation state of ErbB2 is dynamically modulated by its interaction with $\beta 1$-integrins.

Fazekas Z, et al: Two-sided fluorescence resonance energy transfer for assessing molecular interactions of up to three distinct species in confocal microscopy. Cytometry A 2008; 73A(3):209-219. DOI: 10.1002/cyto.a.20489

\section{The Power of One}

Several lines of evidence support the involvement of plasmacytoid pre-dendritic cells (pDCs) in the pathology of human diseases, such as systemic lupus erythematosus, atopic dermatitis and asthma. Thus pDC biology has become a major focus of medical research. The severe setback of pDC-focused studies is the rarity and ex vivo fragility of these cells. Magyarics and co-workers report on a new flow cytometric method using light scatter parameters and one fluorescent dye, only for $\mathrm{pDC}$ identification in peripheral blood samples. Their novel approach may provide a useful tool for the investigation of pDCs in both basic research and clinical routine, as the identification steps leave open two or three further channels for phenotypic and functional analysis of cells by common, two-laser benchtop cytometers.

Magyarics Z, et al: Identification of plasmacytoid pre-dendritic cells by one-color flow cytometry for phenotype screening. Cytometry A 2008;73A(3):254-258. DOI: 10.1002/ cyto.a. 20529

\section{New Cytometric Measurements}

Automatic histological evaluation has been demanded for many years in pathological diagnostics. Several attempts to create such decision support systems - which are able to diagnose certain diseases in certain organs - have been described. 
Earlier, Ficsor and co-workers introduced a technique for gastric biopsy samples which was adequate for the differentiation of normal, gastritis and adenocarcinoma slides (Cytometry B 2006;70B: 423-431). In this issue, they describe the process of cell, gland and epithelium surface analysis on digital slides which play a key role in automated evaluation of colon samples. New tissue cytometric features were created for the characterization of colon diseases by the measurements of main tissue compartments. Further investigations are required to construct novel, organ specific tissue cytometric features for reliable decision support systems in routine pathology.

Ficsor L, et al: Automated classification of inflammation in colon histological sections based on digital microscopy and advanced image analysis. Cytometry A 2008;73A(3):230-237. DOI: 10.1002/cyto.a.20527

\section{Monitoring Your Calcium}

Flow cytometry enables the sequential determination of calcium levels in millions of cells loaded with fluorescent dyes (i.e. the calcium flux) over a period of time. While calcium flux may be of central importance in several biological processes, its analysis with flow cytometry is underused as current algorithms are not able to mathematically handle this large amount of data. A new approach has been developed that characterizes the calcium flux data obtained during lymphocyte activation. First, a five-parametric function was fitted to the medians of data per time unit. With its use the most relevant biological descriptors of the cell activation process (e.g. starting value, peak, time to reach the maximum, the $50 \%$ of maximum before and after the peak) can be estimated. The distribution of data per time unit is characterized by the parameters of functions fitted to percentile values. This approach makes possible the objective comparison of individual calcium flux measurements.

Kaposi AS, et al: Cytometry-acquired calcium-flux data analysis in activated lymphocytes. Cytometry A 2008;73A(3): 246-253. DOI: 10.1002/cyto.a.20518 